\title{
Elisabeth Noelle-Neumann: Kommentar zu Weyma Lübbe
}

\author{
Institut für Demoskopie, D-7753 Allensbach
}

\begin{abstract}
Als Adorno den ersten Entwurf meines Buches „Umfragen in der Massengesellschaft. Einführung in die Methoden der Demoskopie“ gelesen hatte, sagte er, am besten gefalle ihm mein Hinweis, daß die Sprache schon den Unterschied bereithalte zwischen ,jeder“ und ,alle“. Ich hatte damals seine Sensibilität nicht genug gewürdigt, mit der er sofort erkannte, daß man bewußt zwischen den beiden Welten, der individuellen und der sozialen, hin- und hergehen muß, wenn man sich mit Demoskopie beschäftigt. Wenn ich jetzt, dreißig Jahre später, sehe, wie Weyma Lübbe bei ihrer Analyse gefangen bleibt im Denken in Individuen - ,jeder" - und im Rationalismus, wird mir das klar.
\end{abstract}

Mit dem Bewußtsein und dem Verstand des Individuums läßt sich der Prozeß der öffentlichen Meinung nicht erfassen. Alles, was dabei eine Rolle spielt, Isolationsdrohung und Isolationsfurcht, $\mathrm{Ab}$ schätzung von Zunahme und Abnahme von kontroversen Meinungen und Einrichtungen des eigenen Verhaltens darauf hat nichts mit verstandesmäßiger Einsicht zu tun, sondern mit der - man ist versucht zu sagen: vegetativen - sozialen Natur, die jedes Individuum in sich trägt.

Amerikanische Wissenschaftler, die die Theorie der Schweigespirale testen wollten, haben in repräsentativen Umfragen die Frage gestellt: „Empfinden Sie Isolationsfurcht?" Und fast alle Befragten sagten: „Nein“. Lübbe möchte die Personen eines repräsentativen Querschnitts fragen: „Hat sich die öffentliche Meinung verändert?“ Und falls ,ja“: "In welcher Richtung?" Wie sollten das diese Personen beantworten können, da sie nachweisbar häufig nicht einmal über die Veränderung ihrer eigenen Meinungen Auskunft geben können? Jede Panelerhebung zeigt das.

Natürlich ist rational das Äußern einer von der öffentlichen Meinung abweichenden Meinung immer eine legitime Sache. Natürlich macht es rational gar keinen Sinn abzuschätzen, wie die meisten denken, da das von der Demoskopie laufend festgestellt wird. Rational betrachtet ist es sogar illegitim, daß die Gesellschaft Isolationsdrohung ausübt, einen Redner auspfeift, denjenigen, der eine von der öffentlichen Meinung abweichende Meinung öffentlich vertritt, isoliert, die kalte Schulter zeigt, ihn stigmatisiert. Der ganze mächtige Druck, den öffentliche Meinung in der repräsentativen
Demokratie auf das einzelne Mitglied der Gesellschaft und auf die Regierung ausübt, ist, wenn man die gültige Demokratietheorie betrachtet, illegitim.

In der Wirklichkeit aber trägt sich all das zu. Menschen schätzen ab, wie „die meisten“ in einer kontroversen Frage denken, und antworten im Interview ohne Zögern, und ohne Zögern sagen sie auch, wer von zwei Rednern pro und kontra ausgepfiffen wird. Müßte sich nicht auch die Philosophie damit beschäftigen zu erklären, welche Bedeutung diese Realität hat? Zu verstehen, welche Leistungen Menschen, die sich bemühen, sich nicht abweichend von der öffentlichen Meinung zu äußern, für die Gesellschaft erbringen, und ebenso, welche Leistungen diejenigen für die Gesellschaft erbringen, die ihre Isolationsfurcht überwinden und sich abweichend von der öffentlichen Meinung äußern? Müßte sich nicht auch die Philosophie mit dem Thema der sozialen Natur des Menschen beschäftigen, mit dem Bewußtsein von „Öffentlichkeit“, wie es Erving Goffman zum wissenschaftlichen Thema gemacht hat - im Anschluß an Darwin und seine Betrachtungen zum Erröten, das es nur bei Menschen, nicht bei Tieren gebe. Wie ist Gesellschaft möglich? Welche Rolle spielt dabei die Öffentlichkeit, das „public eye“, wie Edmund Burke zuerst formulierte? Um diese Frage zu beantworten, muß die soziale Natur des Menschen mit den Hilfsmitteln der empirischen Sozialforschung, die wir heute haben, verstanden werden.

Wenn ich von der Genauigkeit des „quasistatistischen Organs" spreche (der Ausdruck stammt übrigens von Gerhard Schmidtchen), dann ist gemeint: im Aggregat eines repräsentativen Querschnitts - nicht von ,jedem", aber von allen zusammen - wird nach bisher vorliegenden Erfahrungen nicht nur mit einer gewissen Wahrscheinlichkeit, zum Beispiel 2 zu 1, sondern immer wahrgenommen, welche Seite zu- oder abgenommen hat (von absolut richtigen Schätzungen der Häufigkeitsverteilung ist sowieso nicht die Rede). Unabhängig von bewußter Wahrnehmung durch die Befragten kann man bei wiederholter Messung der öffentlichen Meinung zu verschiedenen Zeitpunkten sehen, wie beide Lager von der stärker gewordenen Seite häufiger als vorher sagen: ,So denken 
die meisten." Und beide Lager bemerken, da $B$ die Gegenmeinung weniger als vorher vertreten wird. Woher kommt diese kollektive Leistung? Was ist ihre Funktion?

Wir wissen bisher viel zuwenig, an welchen Zeichen sich die Menschen bei ihren Urteilen orientieren. Nur die Ursachen verzerrter Vorstellungen über die tatsächliche Häufigkeitsverteilung sind inzwischen weitgehend aufgeklärt durch Inhaltsanalysen von tonangebenden Medien und den auch nachweisbaren Zusammenhang zwischen Medienkonsum und Ausmaß verzerrter Wahrnehmung (,doppeltes Meinungsklima“). ${ }^{1}$

Wir stehen vor einem riesenhaften, wissenschaftlich unbearbeiteten Feld. Das wird mir besonders klar bei den mit leichter Hand hingesetzten Irrtümern von Weyma Lübbe, zum Beispiel: „Wer schweigt, hat nichts einzuwenden." So dachte noch Hobbes, aber heute wissen wir, wenn Menschen zustimmen und glauben, die öffentliche Meinung auf ihrer Seite zu haben, reden sie. Wenn sie schweigen, ist die Wahrscheinlichkeit größer, daß sie nicht zustimmen. Außerdem spielen noch persönliche Anlagen und Fähigkeiten beim Reden

${ }^{1}$ Elisabeth Noelle-Neumann: Das doppelte Meinungsklima. Der Einfluß des Fernsehens im Wahlkampf 1976. In: Politische Vierteljahresschrift. 18, 1977: 408-451 oder Schweigen eine Rolle, das ist ja selbstverständlich. Sogar die Rolle der Leserbriefe in Zeitungen für das Bilden der Vorstellung von öffentlicher Meinung ist wissenschaftlich nachgewiesen worden - entgegen den Annahmen von Lübbe.

Insgesamt stellt sie sich den Prozeß der öffentlichen Meinung zu harmlos vor, als komme es gar nicht so sehr darauf an, ob Menschen diese oder jene Meinung als vorherrschend empfinden. Jeder Proze $ß$ der öffentlichen Meinung drängt dahin, die obsiegende Meinung gesetzlich zu kodifizieren ob es nun um die Anerkennung der Wehrdienstverweigerung oder den Verzicht auf den Jäger 90 oder ein Rauchverbot geht.

Es fällt den Intellektuellen der Gegenwart schwer, den ganzen Ernst und die Kraft des Prozesses der öffentlichen Meinung zu verstehen. Aristoteles hatte klar gesagt: Wenn das Volk den König nicht mehr unterstützt, ist er kein König mehr, und Erasmus von Rotterdam hatte es von ihm übernommen. David Hume hatte festgestellt: Alle Regierung beruht auf Meinung.

Dieses Wissen müssen Philosophen und Politikwissenschaftler zurückgewinnen, und sie müssen versuchen zu verstehen, wie öffentliche Meinung unter Ausnutzung der Isolationsfurcht, der sozialen Natur des Menschen funktioniert, und sie müssen der öffentlichen Meinung in der modernen Welt ihre Legitimität zurückgeben. Hier kann die Demoskopie ihre Aufgabe zur Aufklärung erfüllen. 